\section{Dynamics of animal} movement in an ecological context: dragonfly wing damage reduces flight performance and predation success

\author{
S. A. Combes*, J. D. Crall and S. Mukherjee \\ Department of Organismic and Evolutionary Biology, Concord Field \\ Station, Harvard University, 100 Old Causeway Road, \\ Bedford, MA 01730, USA \\ *Author for correspondence (scombes@oeb.harvard.edu).
}

Much of our understanding of the control and dynamics of animal movement derives from controlled laboratory experiments. While many aspects of animal movement can be probed only in these settings, a more complete understanding of animal locomotion may be gained by linking experiments on relatively simple motions in the laboratory to studies of more complex behaviours in natural settings. To demonstrate the utility of this approach, we examined the effects of wing damage on dragonfly flight performance in both a laboratory drop-escape response and the more natural context of aerial predation. The laboratory experiment shows that hindwing area loss reduces vertical acceleration and average flight velocity, and the predation experiment demonstrates that this type of wing damage results in a significant decline in capture success. Taken together, these results suggest that wing damage may take a serious toll on wild dragonflies, potentially reducing both reproductive success and survival.

Keywords: insect flight; dragonflies; wing damage; predation

\section{INTRODUCTION}

Much of our understanding of the control and dynamics of animal movement derives from controlled laboratory experiments. Laboratory experiments allow investigators to focus on simple movement patterns and to elicit similar behaviours repeatedly, facilitating biomechanical analyses and reducing the impact of individual variability. The repeatability and control provided by laboratory experiments is thus essential for teasing out fundamental principles of animal locomotion. Many of these fundamental principles involve the relationship between locomotory structures (e.g. appendages, muscles, joints) and their functions (e.g. power, control, efficiency); yet, when examined outside of their ecological context, the relevance of

Electronic supplementary material is available at http://dx.doi.org/10. 1098/rsbl.2009.0915 or via http://rsbl.royalsocietypublishing.org.

One contribution of 11 to a Special feature on 'Control and dynamics of animal movement'. structure-function relationships and their significance to wild animals can be difficult to assess.

Linking laboratory analyses of simplified locomotory tasks to studies of natural, complex behaviours can help reveal the ecological relevance and significance of structure-function relationships. This approach has been well developed in studies of terrestrial sprinting and escape behaviour, particularly in lizards (e.g. Irschick et al. 2005), but has rarely been used in studies of animal flight (for an example, see Srygley \& Kingsolver 2000). To demonstrate the utility of this approach for animal flight studies, we examined the effects of wing damage on dragonfly flight performance, in both a laboratory drop-escape response and the more natural context of aerial predation.

Many flying animals suffer episodic (e.g. moulting birds) or cumulative (e.g. insects) loss of wing area, and although the aerodynamic predictions regarding the cost of reduced wing area are straightforward, previous studies investigating the consequences of area loss have yielded conflicting results. In bumblebees (Bombus terrestris), artificial wing wear (approx. $18 \%$ area loss) increases mortality (Cartar 1992). Laboratory experiments show that bees with clipped wings have a higher wingbeat frequency but no increase in metabolic costs, leading to the suggestion that reduced manoeuvreability makes bees with wing damage more susceptible to predators (Hedenström et al. 2001). Increased susceptibility to predators has also been proposed as a consequence of wing area loss in butterflies, as individuals with their hindwings removed (approx. 50\% area loss) fly in equally curvy paths but at a lower overall speed (Jantzen \& Eisner 2008). Yet, an earlier study found that butterflies with clipped wings (15-20\% area loss) display no difference in flight activity, dispersal rates or recapture probability in the wild (Kingsolver 1999).

Thus, the studies performed to date do not demonstrate convincingly that wing area loss carries a functional cost that has a negative impact on fitness, as they lack either a functional explanation (in the case of bumble-bees) or a demonstrated fitness cost (in the case of butterflies). It is important to note that in these studies, fitness has been evaluated only in the context of short-term survival (i.e. evasion of predators over the course of several days), although for many species the need to escape from predators may be a relatively infrequent event as compared with other flight behaviours.

We examined the effects of hindwing area loss-a form of wing damage commonly observed in wild dragonflies-on flight biomechanics during a simple laboratory flight test and during aerial predation. Aerial predation is the only means by which odonates (dragonflies and damselflies) acquire resources, and is a critical component of fitness. The substantial mass gain that results from hunting contributes to flight muscle mass and mating success in males (Marden 1989), as well as to abdominal mass and fecundity in females (Anholt 1991). However, aerial predation is also a risky business: individuals that forage more and gain more weight have a higher mortality rate, presumably owing to the inherent risk of being preyed upon themselves while pursuing a meal (Anholt 1991). Thus, aerial predation is a critical flight behaviour that may have multiple fitness consequences. 
(a)
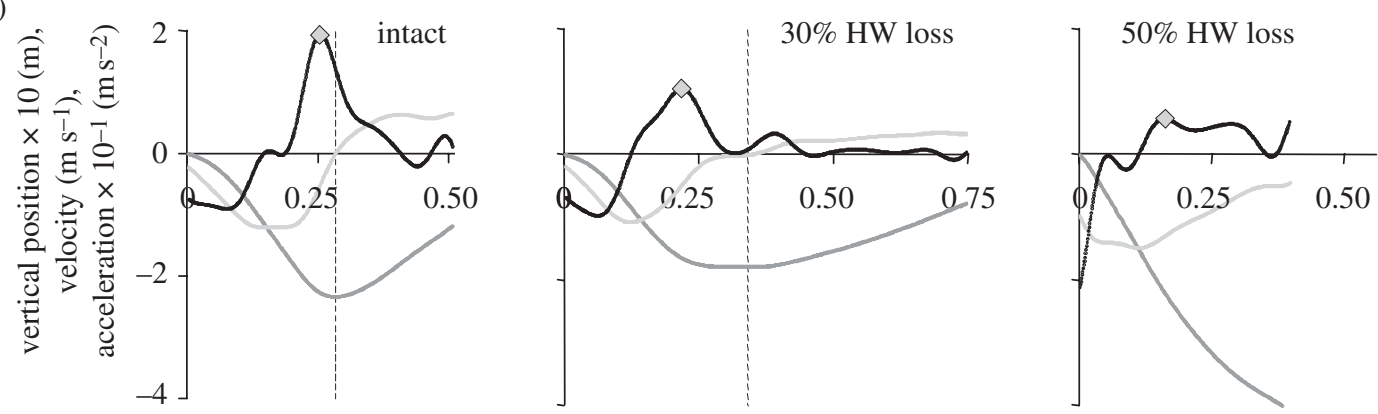

time (s)

(b)

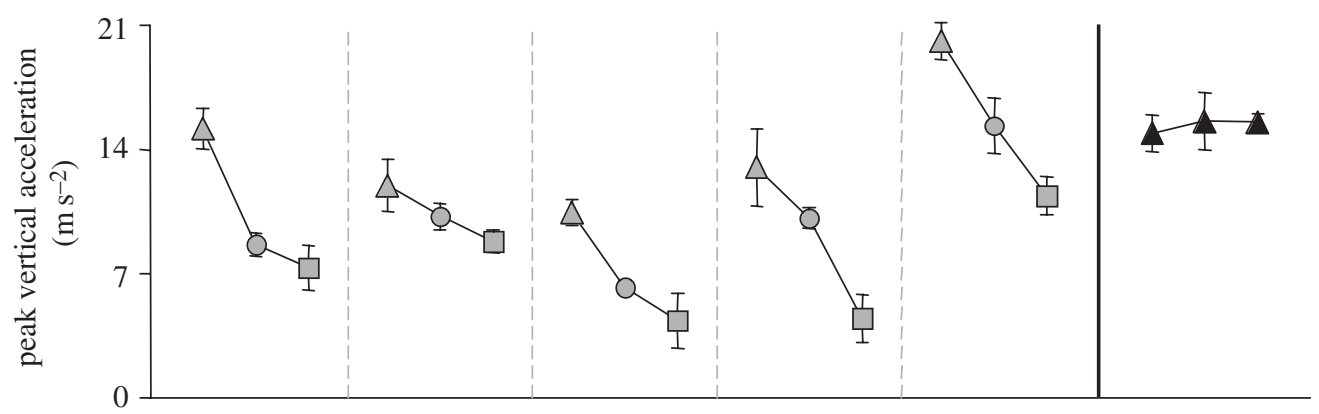

(c)

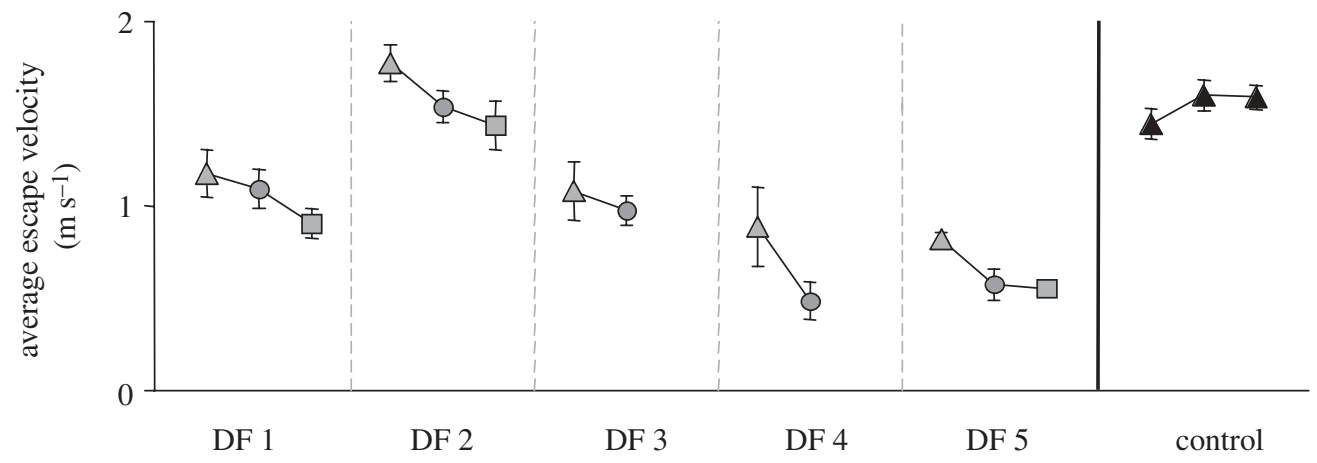

Figure 1. Effects of hindwing area removal on dragonfly flight performance during laboratory drop-escape tests. (a) Sample data from dragonfly no. 4 showing vertical position (dark grey), velocity (light grey) and acceleration (black) versus time with intact wings, 30\% hindwing area removal and 50\% hindwing area removal. Peak acceleration is marked with a diamond, and the end of the drop-start of the escape response is shown with a dashed line. (b) Peak vertical accelerations produced by dragonflies to arrest their fall. (c) Time-averaged velocity during the first $0.15 \mathrm{~s}$ of the escape. Shaded triangle, intact; shaded circle, $30 \%$ hindwing loss; and shaded square, $50 \%$ hindwing loss. Symbols indicate mean \pm s.e. In the control dragonfly, the three symbols represent trials $1-5,6-10$ and $11-15$, respectively.

\section{MATERIAL AND METHODS}

\section{(a) Laboratory experiment: flight performance}

Dragonflies (Sympetrum rubicundulum) were collected in Bedford, MA, in July 2009. Flight performance was assessed during a dropescape response: dragonflies were clasped with wings pressed together and released suddenly from approximately $0.5 \mathrm{~m}$ above a flat surface. Dragonflies typically dropped briefly and then began flapping to stop their descent; after reaching the bottom of the fall, they flew up and away from the point of release.

Flight trajectories of five individuals were recorded using three Photron SA-3 video cameras filming at 1000 frames per second For each dragonfly, five flight sequences were recorded for each of three experimental conditions: (i) fully intact wings, (ii) 30 per cent of hindwing area removed (approx. 18\% total wing area, based on an approx. 60:40 hindwing to forewing area ratio), and (iii) 50 per cent of hindwing area removed (approx. 30\% total wing area). Note that the total wing area removed in the 30 per cent hindwing trials is similar to most previous studies on wing damage, which range from 15 to 20 per cent total area removed in bumble-bees and butterflies. Wing area was removed symmetrically from both hindwings at an oblique angle from the distal leading edge to a more proximal point on the trailing edge, roughly mimicking patterns of wing area loss observed in some older dragonflies. Although the spatial patterns and extent of wing damage in wild insects have not been systematically documented, most major groups of dragonflies (including those used in this study) display natural wing damage ranging from minor tattering to the loss of large wing sections, especially towards the trailing edge and wing tips.

In a few of the laboratory trials, particular sequences could not be analysed owing to atypical flight behaviour or the inability to fly (particularly with $50 \%$ hindwing area removal). We also performed a control experiment, in which one individual with intact wings was subjected to the same handling procedures and performed the same number of flights in the laboratory as experimental individuals, to test for the effects of fatigue or body temperature changes on flight performance.

Body trajectories were digitized using DLTdv3 (Hedrick 2008) and analysed in MAтLAB. Vertical trajectories were filtered using a fifth-order Butterworth filter with a cutoff of $10 \mathrm{~Hz}$ (approx. onethird wing beat frequency), then differentiated sequentially to obtain vertical velocity and acceleration (figure $1 a$ ). In most cases, dragonflies produced a peak in positive vertical acceleration shortly before arresting their fall; this acceleration is beyond that required for weight support (i.e. a hovering dragonfly would have an 

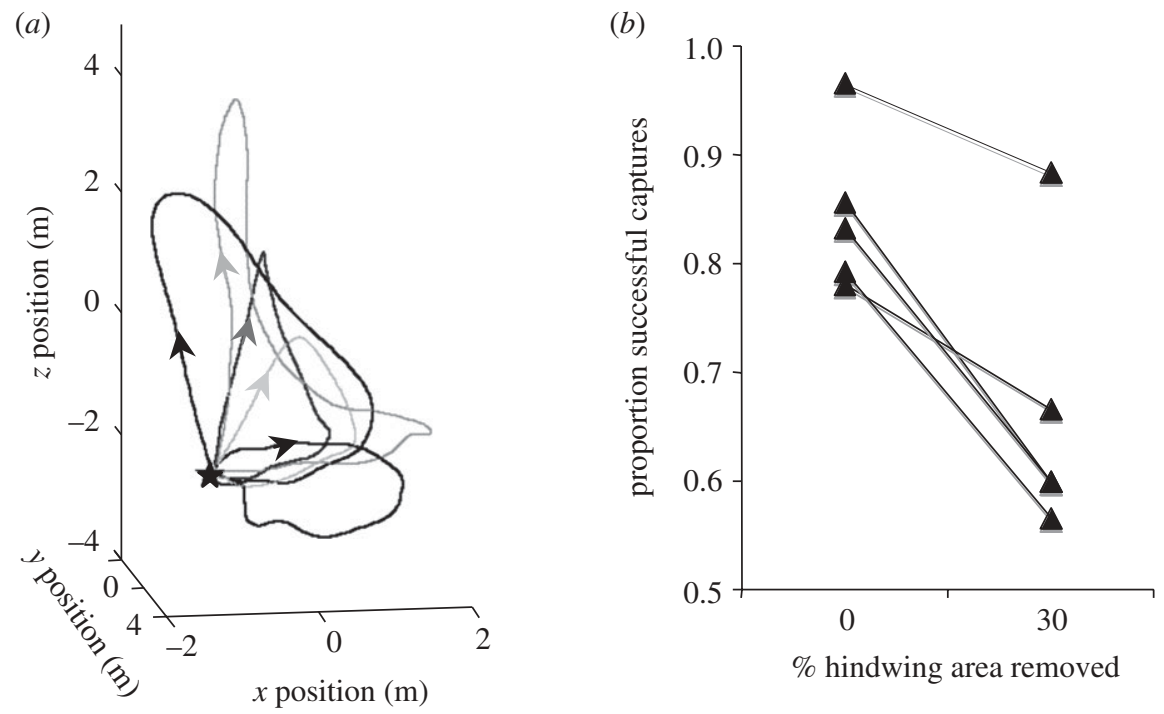

Figure 2. Effect of hindwing area removal on predation success rate. (a) Sample trajectories of predation flights demonstrating the variability and complexity of this behaviour. The star represents the dragonfly's perch, and arrows indicate the direction of flight. (b) Effect of $30 \%$ hindwing area removal on predation success rate in five individuals.

acceleration of $0 \mathrm{~m} \mathrm{~s}^{-2}$ ). In two cases, dragonflies with 50 per cent hindwing area removed were unable to stop their descent in any trial, although they still produced peaks in vertical acceleration (e.g. figure $1 a$, right). In trials where dragonflies did successfully stop their descent and begin to fly away, the total distance travelled during the first $0.15 \mathrm{~s}$ (approx. 4-6 wing beats) after reaching the bottom of the drop was quantified, as this 'escape distance' may be ecologically relevant (e.g. for predator avoidance).

Experimental data were analysed using a two-way analysis of variance (ANOVA) in SPSS, testing for the effects of treatment (wing area loss), individual and treatment $\times$ individual interactions. Data from the control individual were combined into three groups of five trials each (trials $1-5,6-10$ and 11-15), and differences between groups were assessed using a Friedman test.

\section{(b) Greenhouse experiment: predation success}

Dragonflies (Sympetrum vicinum) were collected in Bedford, MA, in late August and September 2009. Individuals were numbered and released into an outdoor, screened greenhouse enclosure (approx. $7.3 \times 7.3 \times 4.6 \mathrm{~m}$, open and equilibrated to the outside environment) with greenery, ponds and perching sticks, where they were allowed to acclimate for at least one day before trials began, feeding on naturally occurring prey. To measure predation success rate, fruit flies (Drosophila melanogaster) were released in front of perched dragonflies; the predation attempt was considered successful if mastication (which occurs over a far longer time scale than aerial predation) was observed after the dragonfly returned to its perch.

Over the course of several days, prey capture success rates were measured first with fully intact wings, then with 30 per cent of hindwing area symmetrically removed. Five individuals completed a full series of capture experiments, engaging in approximately 30 predation attempts before and after wing clipping $\left(\bar{n}_{\text {intact }}=31 ; \bar{n}_{\text {clippped }}=27\right)$. The change in capture success rate was evaluated in two ways. First, a $\chi^{2}$-test of homogeneity was used to determine whether the proportions of captures and failures for each individual after wing clipping were significantly different from those expected, based on each individual's success rate with intact wings. This test accounted for the varying numbers of trials conducted for each individual/treatment combination. A more conservative Wilcoxon signed-rank test for paired samples was also performed, evaluating the difference between overall success rate before and after wing clipping.

\section{RESULTS}

\section{(a) Laboratory experiment: flight performance}

Peak vertical accelerations generated by dragonflies to stop their descent declined significantly with hindwing area removal (two-way ANOVA: treatment, $p<$ 0.0001 ; individual, $p<0.0001$; treatment $\times$ individual, $p=0.19$; figure $1 b$ ). Removing 30 per cent of hindwing area (only $18 \%$ of total wing area) caused vertical acceleration to drop by nearly 30 per cent $(\bar{x}=28.7 \pm 12.3 \%)$, while 50 per cent hindwing area removal (30\% total wing area) caused a drop in vertical acceleration of nearly 50 per cent $(\bar{x}=49.1 \pm 15.2 \%$; table $S 1$ in the electronic supplementary material). In cases where dragonflies were able to stop their fall and initiate an escape trajectory, time-averaged velocity also declined significantly with hindwing area removal (two-way ANOVA: treatment, $p=0.001$; individual, $p<0.0001 ; \quad$ treatment $\times$ individual, $\quad p=0.815$; figure $1 c$ ); escape velocity declined by 21.0 per cent $( \pm 7.2 \%)$ with 30 per cent hindwing area removal and by 24.8 per cent $( \pm 3.9 \%)$ with 50 per cent hindwing area removal (table $\mathrm{S} 1$ in the electronic supplementary material). In contrast, neither vertical acceleration nor average escape velocity differed between the three sequential groups of trials in the control dragonfly (Friedman test: vertical acceleration, $p=0.819$; escape velocity, $p=0.4491$; table $\mathrm{S} 1$ in the electronic supplementary material).

\section{(b) Greenhouse experiment: predation success}

The trajectories of hunting dragonflies were far more complex and variable (figure $2 a$ ) than the trajectories elicited in the laboratory drop-escape tests (figure $1 a$ ), and generally included a large vertical component preceding prey capture. Capture success rate declined in all five individuals after 30 per cent of hindwing area was removed $(\bar{x}$ decline $=21.9 \pm 9.7 \%$; figure $2 b$ ). The proportions of captures and failures after wing trimming differed significantly from expectations based on each individual's intact success rate $\left(\chi^{2}\right.$-test of homogeneity: $p<0.0001$ ), and overall capture rates were significantly different before and after wing trimming (Wilcoxon signed-rank test: $p=0.043$ ).

\section{DISCUSSION}

Taken together, the laboratory and greenhouse experiments suggest that hindwing damage may take a 
serious toll on wild dragonflies: wing area loss reduces both vertical acceleration and flight velocity, and compromises predation success. This story would be far less complete if either experiment had been performed in isolation. The simple trajectories elicited in the laboratory allowed us to quantify biomechanical (functional) traits affected by wing damage that would have been difficult to isolate from complex predation trajectories, and the repeatability of the drop-escape test allowed us to identify trends despite the high variability between and within individuals. The greenhouse experiment allowed us to determine the relevance of these findings by demonstrating that wing damage results in reduced predation success, suggesting that the biomechanical variables identified in the laboratory may be important contributors to predation success in the wild.

Damage involving the loss of a significant portion of the wing is likely to cause a number of detrimental effects, beyond simply the loss of aerodynamically active wing area. Most insects possess sensory hairs (sensillae) on the surfaces of their wings, and thus the loss of wing area also results in the loss of sensory information, which may affect flight control. In addition, the loss of wing area may result in wing drying or other physical changes in the remaining wing portion. Finally, changes in wing shape resulting from various patterns of wing damage may affect aerodynamic force production, independent of the amount of area that is lost. While future experiments are planned to address the latter issue, it is unfortunately impossible to remove wing area without the associated loss of wing sensors and potential drying effects. Thus, these additional factors (which also occur in response to natural wing damage) may contribute to the changes in flight performance observed.

How might the reduced capture success observed in dragonflies with wing damage affect their fitness? Because the proportion of unsuccessful predation attempts increases as capture success declines, less successful dragonflies are expending more energy on flight per unit of energy they gain through prey capture. This is likely to limit the amount of body mass they can gain, lowering fecundity and/or mating success (Marden 1989; Anholt 1991). In addition, if less successful hunters engage in more pursuits, in an attempt to compensate for their lower success rate, they may experience higher mortality owing to increased exposure to aerial predators (Anholt 1991).
Although a direct demonstration of these consequences awaits field studies on natural populations of dragonflies, the results of our combined laboratory and greenhouse experiments suggest that wing damage, a common occurrence in many groups of insects, may have significant fitness consequences. Thus, overall wing morphology is likely to reflect not only selection for effective aerodynamic force generation, but also for wing durability - the ability of wings to undergo numerous flapping cycles and collisions while avoiding or minimizing structural damage.

The authors would like to thank Ryan Neely for help digitizing videos and Ty Hedrick for the use of his digitizing software.

Anholt, B. R. 1991 Measuring selection on a population of damselflies with a manipulated phenotype. Evolution 45, 1091-1106. (doi:10.2307/2409718)

Cartar, R. V. 1992 Morphological senescence and longevity: an experiment relating wing wear and life span in foraging wild bumble bees. F. An. Ecol. 61, 225-231. (doi:10. 2307/5525)

Hedenström, A., Ellington, C. P. \& Wolf, T. J. 2001 Wing wear, aerodynamics and flight energetics in bumblebees (Bombus terrestris): an experimental study. Funct. Ecol. 15, 417-422. (doi:10.1046/j.0269-8463.2001.00531.x)

Hedrick, T. L. 2008 Software techniques for two- and threedimensional kinematic measurements of biological and biomimetic systems. Bioinsp. Biomim. 3, 034001. (doi:10.1088/1748-3182/3/3/034001)

Irschick, D. J., Herrel, A., Vanhooydonck, B., Huyghe, K. \& Van Damme, R. 2005 Locomotor compensation creates a mismatch between laboratory and field estimates of escape speed in lizards: a cautionary tale for performance-to-fitness studies. Evolution 59, 1579-1587.

Jantzen, B. \& Eisner, T. 2008 Hindwings are unnecessary for flight but essential for execution of normal evasive flight in Lepidoptera. Proc. Natl Acad. Sci. USA 105, $16636-$ 16 640. (doi:10.1073/pnas.0807223105)

Kingsolver, J. G. 1999 Experimental analyses of wing size, flight, and survival in the western white butterfly. Evolution 53, 1479-1490. (doi:10.2307/2640894)

Marden, J. H. 1989 Bodybuilding dragonflies: costs and benefits of maximizing flight muscle. Phys. Zool. 62, $505-521$.

Srygley, R. B. \& Kingsolver, J. G. 2000 Effects of weight loading on flight performance and survival of palatable Neotropical Anartia fatima butterflies. Biol. F. Linn. Soc. 70, 707-725. (doi:10.1111/j.1095-8312.2000.tb00225.x) 